\title{
Improved Energy-Efficient Algorithm in Wireless Communication Systems using PSO Method
}

\author{
Samira Achki and Fatima Gharnati \\ Computer Science Department \\ Faculty of Science Semlalia, Cadi Ayyad University, Marrakech, Morocco \\ S.ichki@gmail.com andf.gharnati@uca.ma
}

\begin{abstract}
Wireless cellular communications have seen an increased development in last years. With the appearance of the smartphone, the desire for higher data rates has grown rapidly, for that Energy efficient EE of wireless systems, communication is an important goal among designed wireless cellular networks, which received more attention. Recently, researchers provide a renewed focus on energy-efficiency in wireless networks that comes from the different perspectives of reducing. In this paper, we are interested in reducing the energy consumption using a new algorithm, which focuses on several parameters such as mobility, distance, location and the base station selection. Therefore, our objective is to locate each user after detection in the BS area, taking into account its speed using the particle optimization algorithm (PSO) for minimizing the energy consumed at each user. The position estimation at several moments in the cell allows an important maximization of the energy efficiency that was proven by the simulation results.
\end{abstract}

Keywords: Ennergy Effeceint; algorithm PSO; wirless cellular network; base station

\section{Introduction}

Due to the development technologies increased, wireless communications have been designed in order to response to different needs especially in our daily lives, such as a huge number of different mobile communications[1]. Several mobile applications, created also to ensure needs of users in all the world and considering all areas, like social media, education, monitoring, health care, sport, etc. While all these applications offer services, they required more energy consumed during using. Where, energy is the first critical limitation in the world of wireless communications.

For that, several research works have been invented in the last years to overcome this problem and to always give to users the best of things.

Hardware proposal scheme and Protocols designs are some big area of interest of research development in communication systems, which allowed enhancing energy consumed and extending life times of mobiles. Despite all inventions, the problem of energy remains a barrier to any technology. Several projects such as Green Radio and EARTH[2] and eWin[3] and OPERA-Net[4], confirm that the BS is the most network element, which consumes more energy.

The first project focus on enhancing the energy metrics and models and the energy efficiency hardware and architectures and the management of EE resource. The second project aims at optimizing the architectures and managing the resource energy. Moreover, It allows the enhancement of the energy metrics and targets and radio technologies and components. The third project improved the $\mathrm{EE}$ architectures and the resource management. The last one considered the link level and technology enablers and the network test bed.

Received (June 15, 2017), Review Result (September 23, 2017), Accepted (November 20, 2017) 
In literature, we have different approaches that resolve the increasing of energy consumption at BS as shown Figure 1. Among these solutions, the cross layer optimization [5] and the switching off strategy[6] and beam forming technique[7]. However, they did not take into account the user mobility.

For that, we proposed new scheme called Smart new PSO application for predicting the next new user position. That allows optimizing energy. The PSO approach used will be explained with more details in section three.

Our paper covers four sections, the second one, is considered for related works, the third one describes the conceptual model and proposed work and the last one, is considered for the simulations and results and their discussion.

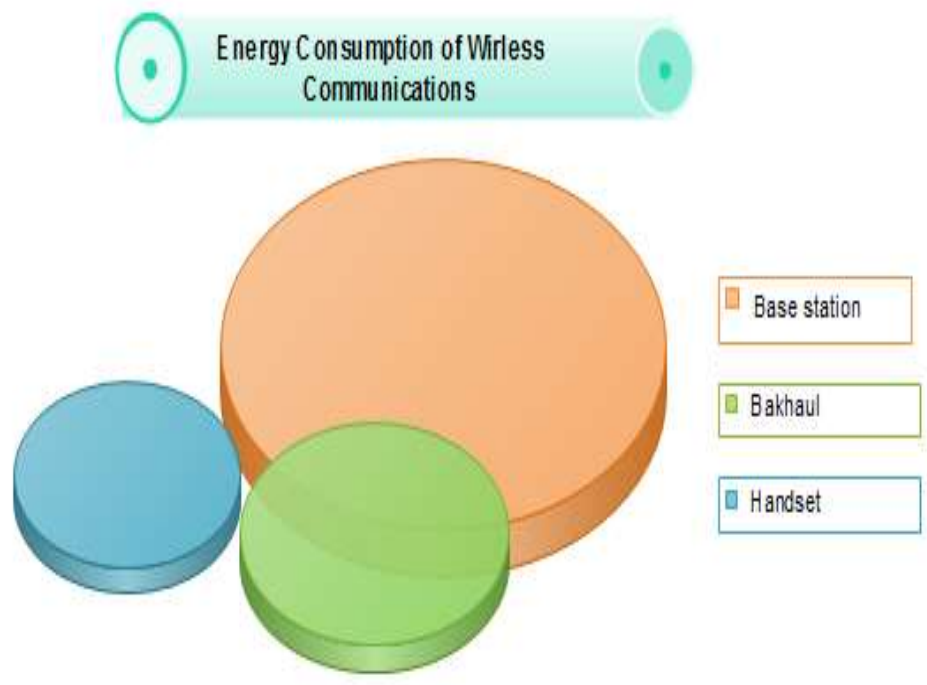

Figure 1. Cellular Network Ennergy Consomption

\section{Related Work}

This section summarizes the popular studies done in wireless communication systems for saving energy:

$\operatorname{In}[8]$, a new algorithm is designed for reducing energy in cellular networks, this novel algorithm is based on these steps: the first one is selecting a user (US) randomly, the next step aims to sort the active BS in increased way. The third step is picking the first one in the sorted list. The last step focus on selecting the followed US for until all users are picked.

In [9] , authors proposed a novel tool called Energy Box that give us the opportunity to estimate the energy spent in several devices, due to the traffic pattern while exploiting the $3 \mathrm{G}$ or WIFI communication. This study has proven a significant enhancement of $99 \%$. Moreover, the aggregation concept was exploited in [10] in order to calculate the energy drained while using the smartphone. This new approach permits collecting the active data while using phones for enhancing the network lifetime.

In [11], authors proposed a novel management system called PhoneJoule. This system is performed using different modes and switching between different communication systems such as WI-FI and GPRS for saving energy. Thanks to this new system, the management of the device battery becomes easier and it has been proven that the energy is reduced in smartphone.

Therefore, there are several mobility models that are designed for the user mobility management. Among these models, the model described in [12]. This model is based on analyzing the mobile user time using the Markovien characteristics. It allows determining 
the probability of the presence of a user at known position. In this model, each user moves according to the markovien process assuming that it uses the simple case known as Brownian motion. The probability of finding user is expressed using its time $t$ and location $\mathrm{r}$, as shown in the following equation:

$$
P\left(r, t ; r_{0}, 0\right)=e^{-M_{0} t}\left(r, r_{0}\right)
$$

Where M0: represents the operator of the generation.

Mobility is an important factor of wireless networks. It gives UEs freedom of movement. Based on the radio technology, we have two mobility limites. It is limited to pedestrian speeds only, or can support communication even at high speeds [13]. We can categorize the mobility concepts in two different key concepts: roaming and handovers.

Roaming represents the movement of a UE from one network to another while handover is the process of switching a call or session that is in progress from one physical channel to another [14].

\section{Our Proposed}

Our paper aims at integrating the PSO algorithm for localizing the users efficiently. This integration allows assigning the users to the BS for easing the user selection. In this work, we take into account the distance separating the user and the active BSs using the user velocity. The next sub-section will detail the operating process of the PSO method.

We considered a simple scenario as shown Figure 2 where a user or device at mobile situation. It can communicate with the BS through relaying its information via other devices at the edge of a cell or in a poor coverage area.

Hence, users can realize a higher QoS or maximize their battery life.

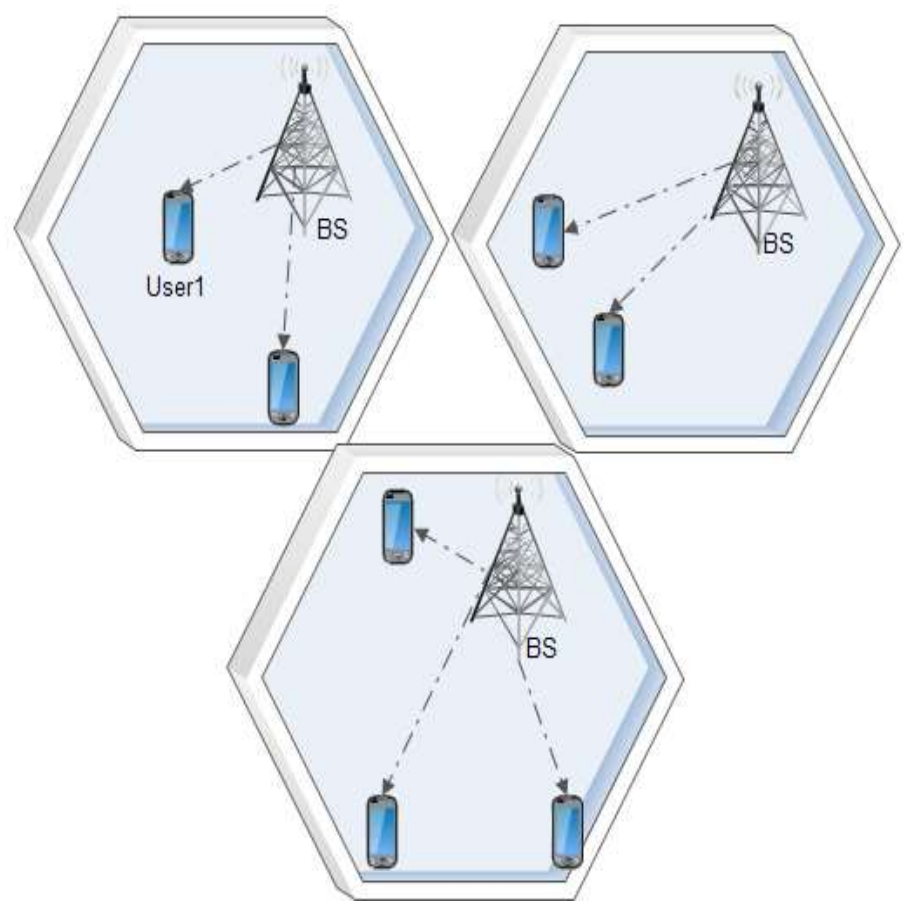

Figure 2. Architecture of Wirless Communication 


\section{A. Pso Method}

PSO method has been inspired from the bird behavior for searching its food. The bird followed the element (bird) that is near to food. The birds are grouped for forming what we call swarms. The main goal of PSO is finding the best solution through the particles number [15]. These particles are able to interact with the neighborhood. The neighborhood selection defined the swarm topology that can be categorized in two well known topologies as shown Figure 3:

- Star topology: each particle is able to communicate to other particle belonging to the swarm. This topology kind is characterized by its fast convergence.

- Ring topology: the communication can be between particles and two neighbors. Unlike the previous topology, this kind has a slow convergence.

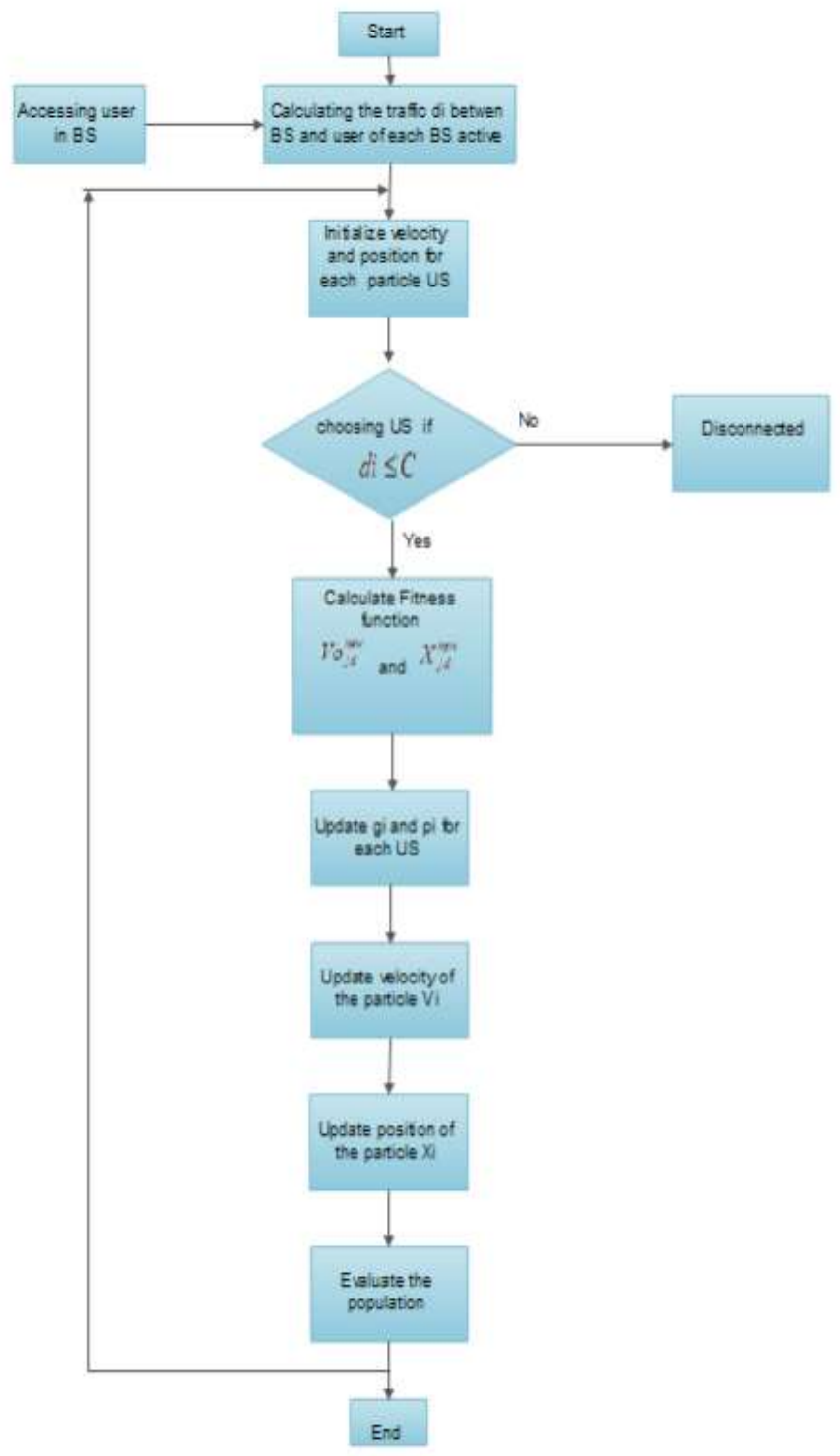

Figure 3. A Flow Chart of the Proposed Algorithm SWARM

In PSO, particles change their localization exploiting a number of known parameters such as: position and velocity and distance. 
The PSO fitness function depends on the nature of the problem studied. PSO equations are described s follows:

$$
\begin{gathered}
V o_{j d}^{\text {new }}=V o_{j d}^{\text {old }}+\mu 1 * \alpha *\left(\operatorname{Pr}_{j d}-X_{j d}\right)+\mu 2 * \beta *\left(\operatorname{Pr}_{g d}-X_{j d}\right) \\
X_{j d}^{\text {new }}=X_{j d}^{\text {old }}+V o_{j d}^{\text {new }}
\end{gathered}
$$

Where:1

$V o_{j d}^{\text {new }}$ : the particle velocity in dimension d.

$X_{j d}$ : the particle position in dimension d.

$\mu 1 \mu 2$ : constants values.

$\alpha \beta$ : Values picked randomly.

$\operatorname{Pr}_{j d}$ : the most efficient localization obtained far by the particle.

$\operatorname{Pr}_{g d}$ : the global efficient localization obtained by the neighborhood.

The PSO process is based on initializing the position and velocity of particles in random way. After that the step of evaluation begins where all fitness values of particles are evaluated, then modify it if the value is more efficient than the best individual fitness. The followed step is obtaining the new position and velocity after updating the global fitness value.

\section{B. Power Consomption Model}

The power model of macro BS detailed in [18] represents a linear relationship between average radiated power per site and average power consumption. We can modify the power consumption to be varied with the traffic load level and the BS. We can express the consumed power Pcon by the BS $\mathrm{j}$ using the following formula:

$$
\text { Pcon }=l \cdot \text { NscNat }(\mathrm{APant}+\mathrm{B})
$$

where

$l \in[0,1]$ is the load factor

Nsc denotes the BS's number of sectors

Nat represents the number of antennas per sector.

Pcon: denotes the total power per BS.

Pant: the power fed to the antenna.

A: represents a coefficient which determines that there is a proportional relation between the power consumed to the power of transmission.

$\mathrm{B}$ denotes the power that is consumed independent of the average transmits power and models the power consumed (e.g., signal processing, site cooling, backhaul, and as well as a battery backup). The previous coefficient has constant values for macro BS. The power model aims to calculate the power consumed considering the power of transmission Ptx. It is obviously clear that this assumption is valid due to the strong dependence of the power consumed at macro sites and the traffic load [19]. The value of the parameter $L$ represents the activity level of the BS that allows describing the portion of resources allocated for transmission task, where the no activity of user in current cell is expressed by the value zero and and the resources availability of one or different users represents the full load. Networks with different site densities are characterized by their different coverage. Hence, it is not suitable to compare them considering only the power consumption.

To model the power consumption in real constraints, the area power APC is introduced as essential parameter that is measured in [W/km2]. It is calculated using the following formula [20], [21]: 


$$
A P C=\frac{P C}{A_{B S}}
$$

Where:

Pc: represents the total power consumed and

Ass is the reference area.

The second value is calculated by the following formula [23]:

$$
A_{B S}=\frac{3 \sqrt{3}}{2} d^{2}
$$

In order to optimize the cell coverage, the area deployed as hexagonal.

The power consumption is increased with two criterion distance and mobility, If the distance is increased the power consumption increase show Figure 4.

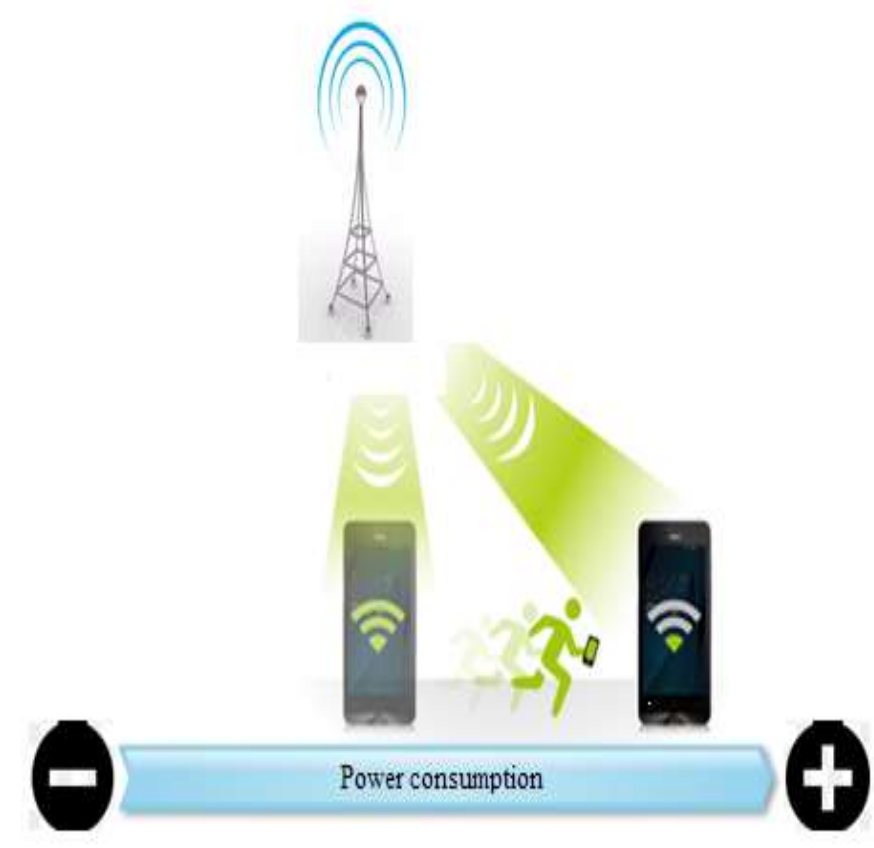

Figure 4. Communication Power vs Distance

\section{Ennergy Effeceicy}

Many works reveal that it is mandatory to enhance the LTE energy efficiency for providing a significant growth on wireless communications. Hence, we consider that the total network energy efficiency EE is calculated by dividing the total amount data delivered by the total power drained. The measure of this ratio is bits per Joule[22]:

$$
\mathrm{ET}=\frac{\sum_{j=1}^{N B S} R j}{\sum_{j=1}^{N B S} \operatorname{Pcoj}}
$$

where

Pc represents the power consumption

$\mathrm{Rj}$ : represents is the total data rate with a BS.

NBS : represents the total number of BS. 


\section{Result and Discusion}

The proposed Simulator MATLAB used for designing the network scenario and evaluating the performances of our approach.

The simulation procedure and system parameters are discussed. The parameters that are affecting the cell size and EE, in simulation, we use a case of cell BS where the cell radius is 500 meters. The bandwidth of BS is $2,6 \mathrm{MHz}$. The traffics of US obey uniform distribution with traffic rates varying within the range $128 \sim 512 \mathrm{kbps}$. BS covers a hexagonal shaped area as shown in Figure 2 where $\mathrm{R}$ is the cell radius and ABS is the coverage area, The cell size is determined according to the minimum received power level constraints. The receiver sensitivity is calculated based on sufficient SINR for the specified,

$$
S I N R=\frac{S_{i p}}{I+N}
$$

Where :

Sip: indicates the power of measured usable signals.

I: indicates the average interference power : the power of measured signals or channel interference signals from other cells in the current system.

$\mathrm{N}$ : indicates background noise, which is related to measurement bandwidths and receiver noise coefficients

The received SNR is calculated based on the received power level and white noise which are estimated according to the path loss model described in 3GPP[23].Then, the achievable data rate within each BS's coverage area is determined based on the SNR distribution in the cell. The power consumption models consist of dynamic power consumption, which is fully depended on the traffic load as.

\section{A. Simulation Scenario}

In general, the cell is controlled by a transmitter/receiver called base station (BS) as shown Figure 3 that provides the radio link with UEs in its coverage area. The coverage of a base station is limited by several factors including:

- The transmit power of UE and BS;

- The radio technology used.

- The wave propagation in the environment (urban, rural, etc.);

- The frequency used;

- The type of antennas used for BS and UE; 

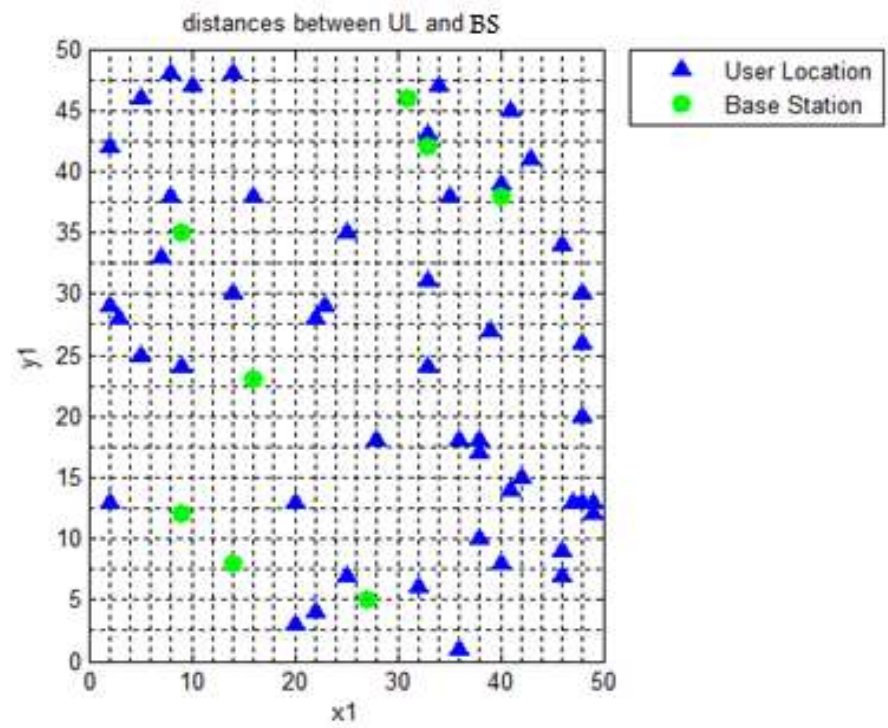

Figure 5. Simulation Scenario

The simulation procedure is done in Matlab. The parameters that we use are summarized in Table I, Constant energy cost factor is derived from the report of power consumption in RRC CONNECTED state of UEs moving with discontinuous reception period.

The power consumption models consist of dynamic power consumption which is fully depended on the traffic load as expressed in Equation. [12]

The simulation parameters are based on 3GPP macrocell model with a system bandwidth of $10 \mathrm{MHz}$ with UE height of $1.5 \mathrm{~m}$.

Table 1. Table of Parameters and Values

\begin{tabular}{|c|c|}
\hline Parameter & Value \\
\hline Number of antennas & $\mathbf{2}$ \\
\hline Number of Sectors & MATLAB \\
\hline simulators & $\mathbf{1 0 ~ M H z}$ \\
\hline Bandwidth frequency & $\mathbf{5 0 0 m}$ \\
\hline Coverage degree & $\mathbf{4 6}$ \\
\hline Transmit power [dBm] & $\mathbf{9}$ \\
\hline Noise figure [dB] & $\mathbf{3}$ \\
\hline $\begin{array}{c}\text { Diversity gain[dB] } \\
\text { Power consumption } \\
\text { parameters } \\
\text { [W] }\end{array}$ & $\mathbf{2 1 . 4 5}$ \\
\hline \begin{tabular}{c} 
Carrier frequency[GHz] \\
\hline
\end{tabular} & 2,6 \\
\hline
\end{tabular}




\section{B. Results}

To evaluate the performance of EE-PSO approach in system communication, simulations are carried out using the parameters shown in table above.

When applying the algorithms PSO above to solve the fitness function, we can obtain their numerical results as shown in Figures below:

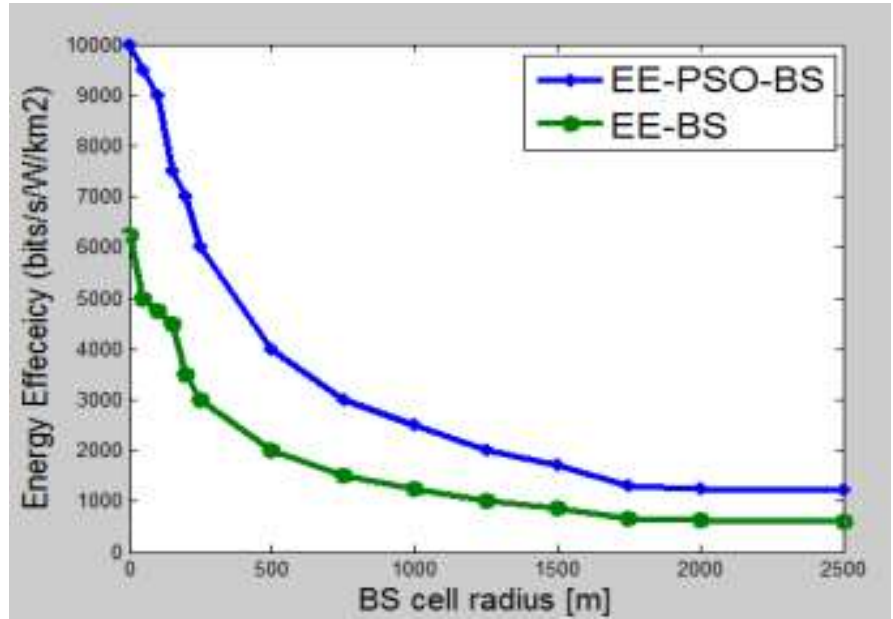

Figure 6. EE for Cell BS

It is clearly shown from Figure 6 that our approach presents the better values in terms of energy efficiency comparatively to the other approaches. This is due to the use of the PSO method, which allows predicting the future user position and selecting the most adequate $\mathrm{BS}$ for communication.

Selecting the BS efficiently according to the current position has permit improving the energy efficiency of our new approach because it eases the control of the signal and reduces the user power.

In Figure 7, we present the results of the power consumption for BS cell which compared between two results. The first one is power consumption without PSO method and the second is our approach based on PSO method, Therefore, it can be concluded that the power consumption is decreased when the PSO method is used with respect to the normal power consumption.

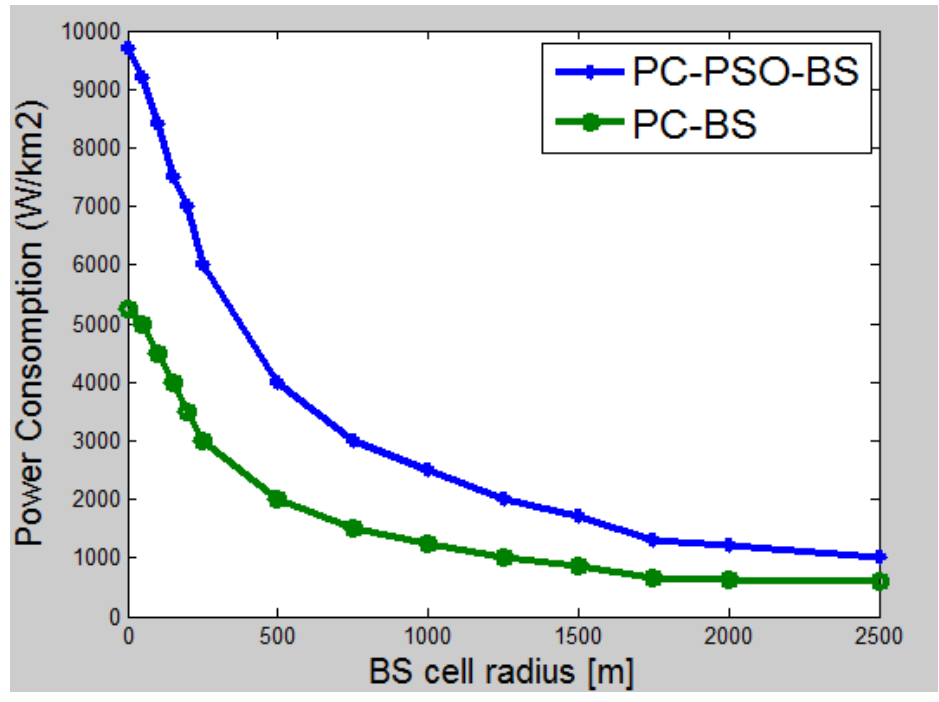

Figure 7. Power Consumption For BS Cell 


\section{Conclusion}

Our daily needs summarized in high mobility provide the appearance of the energetic problem. This push researchers to make real efforts for realizing this great task.

Different works have been designed to reduce the energy consumption of the wireless systems. However, few of them consider the user mobility in their conceptual models or in communication phases. In this paper, we intend to improve the energy efficiency using the PSO algorithm. Our novel algorithm permits predicting the next position and selecting the efficient BS, which reduced the energy consumed by users. Simulation results have shown that our approach is more efficient that the previous methods.

\section{Acknowledgment}

This work is supported by University Cadi ayyad Semlalia.

\section{References}

[1] A. Samira, F. Gharnati and A. A. Ouahman, "Enhancing Energy Consumption in Wireless Communication Systems using Weighted Sum Approach", Indian Journal of Science and Technology, http://dx.doi.org/10.17485/ijst\%2F2017\%2Fv10i4\%2F110903, vol. 10, issue 4, (2017).

[2] EARTH, "Energy aware radio and network technologies project," https://www.ict-earth.eu/default.html. I.S. Jacobs and C.P. Bean, "Fine particles, thin films and exchange anisotropy", in Magnetism, Eds. New York: Academic, vol. III, (1963), pp. 271-350.

[3] "Energy efficiency enhancements in radio access networks", Wireless@KTH Research Strategy Document 2008-2010, Wireless@KTH, http://www.wireless.kth.se/images/stories/] Strategy/Research plan08.pdf, (2008).

[4] "Optimising power efficiency in mobile radio networks", OPERA-Net PROJECT STAND \# 42, 2010 NEM SummitTowards Future Media Internet, Barcelona,Spain, Oct 2010, http://operanet.org/Documents/5026v1 Opera-Net e-NEM\%20Event\%20Barcelona\%202010 Demos\%20Presentation 290710.pdf.

[5] W. Su and T. L. Lim, "Cross-layer design and optimisation for wireless sensor networks", International Journal of Sensor Networks, http://doi.org/10.1504/IJSNet.2009.028021, vol. 6, no. 1, (2009), pp. 3-12.

[6] M. Feng, S. Mao and T. Jiang, "BOOST: Base station ON-OFF switching strategy for energy efficient massive MIMO HetNets", In Proceedings - IEEE INFOCOM, http://doi.org/10.1109/INFOCOM.2016.7524485, vol. 2016, (2016).

[7] B. Li, Z. Zhou, W. Zou, X. Sun and G. Du, "On the efficient beam-forming training for $60 \mathrm{GHz}$ wireless personal area networks", IEEE Transactions on Wireless Communications, http://doi.org/10.1109/TWC.2012.121412.110419, vol. 12, no. 2, (2013), pp. 504-515.

[8] K. Shen and W. Yu, "Distributed pricing-based user association for downlink heterogeneous cellular networks", IEEE Journal on Selected Areas in Communications. http://doi.org/10.1109/JSAC.2014.2328143, vol. 32, no. 6, (2014), pp. 1100-1113.

[9] W. H. Yang, Y. C. Wang, Y. C. Tseng and B. S. P. Lin, "Energy-efficient network selection with mobility pattern awareness in an integrated WiMAX and WiFi network", International Journal of Communication Systemshttp://doi.org/10.1002/dac.1066, vol. 23, no. 2, (2010), pp. 213-230.

[10] Y. Jiang, X. Pan, K. Li, Q. Lv, R. P. Dick, M. Hannigan and L. Shang, "ARIEL: Automatic Wi-Fi based Room Fingerprinting for Indoor Localization", Proceedings of the 2012 ACM Conference on Ubiquitous Computing. http://doi.org/10.1145/2370216.2370282, (2012), pp. 441-450

[11] P. García, J. P. Torreglosa, L. M. Fernández and F. Jurado, «Optimal energy management system for stand-alone wind turbine/photovoltaic/hydrogen/battery hybrid system with supervisory control based on fuzzy logic", International Journal of Hydrogen Energy, http://doi.org/10.1016/j.ijhydene.2013.08.106, vol. 38, no. 33, (2013), pp. 14146-14158.

[12] Q. Li, Y. Zheng, X. Xie, Y. Chen, W. Liu and W. Y. Ma, « Mining user similarity based on location history", Proceedings of the 16th ACM SIGSPATIAL International Conference on Advances in Geographic Information Systems http://doi.org/10.1145/1463434.1463477,no. (c), (2008), p. 34.

[13] C. Makaya and S. Pierre, "An architecture for seamless mobility support in IP-based next-generation wireless networks", IEEE Transactions on Vehicular Technology, http://doi.org/10.1109/TVT.2007.906366, vol. 57, no. 2, (2008), pp. 1209-1225.

[14] M. Mahedi Hassan and P. K. Hoong, "Seamless handover integrated solution for video transmission over proxy mobile IPv6 in a micro mobility domain", Journal of Network and Computer Applications. http://doi.org/10.1016/j.jnca.2012.04.020, (2013).

[15] A. I. Selvakumar and K. Thanushkodi, "Anti-predatory particle swarm optimization: Solution to nonconvex economic dispatch problems", Electric Power Systems Research, http://doi.org/10.1016/j.epsr.2006.12.001, vol. 78, no. 1, (2008), pp. 2-10.. 
[16] M. Li, D. Lin and J. Kou, «A hybrid niching PSO enhanced with recombination-replacement crowding strategy for multimodal function optimization", Applied Soft Computing Journal, https://doi.org/10.1016/j.asoc.2011.11.032, vol. 12, no. 3, (2012), pp. 975-987.

[17] X. Jin, Y. Liang, D. Tian and F. Zhuang, "Particle swarm optimization using dimension selection methods", Applied Mathematics and Computation, https://doi.org/10.1016/j.amc.2012.11.020, vol. 219, no. $10,(\mathbf{2 0 1 3})$, pp. $5185-5197 .$.

[18] L. Zhang, B.Tiwana, Z.Qian, Z. Wang, L.Zhangt, R. P. Dickt and L. Yang, "Accurate online power estimation and automatic battery behavior based power model generation for smartphones", Proceedings of the, https://doi.org/10.1145/1878961.1878982, (2010), pp. 105-114.

[19] A. Coiro, M. Listanti, A. Valenti and F. Matera, «Reducing power consumption in wavelength routed networks by selective switch off of optical links", IEEE Journal on Selected Topics in Quantum Electronics, https://doi.org/10.1109/JSTQE.2010.2052791, vol. 17, no. 2, (2011), pp. 428-436.

[20] S. N. Shahab, T. S. Kiong and A. A. Abdulkafi, "A framework for energy efficiency evaluation of LTE network in urban, suburban and rural areas", Australian J. Basic Appl. Sci., vol. 7, no. 7, (2013), pp. 404-413.

[21] S.N. Shahab, A. A. Abdulkafi and A. R. Zainun, "Assessment of Area Energy Efficiency of LTE Macro Base Stations in Different Environments", Journal of Telecommunications and Information Technology, no. 1 , (2015), p. 59

[22] D. W. K. Ng, E. S. Lo and R. Schober, "Energy-efficient resource allocation in multi-cell OFDMA systems with limited backhaul capacity", IEEE Transactions on Wireless Communications, https://doi.org/10.1109/TWC.2012.083112.111951, vol. 11, no. 10, (2012), pp. 3618-3631.

[23] 3GPP TR 36.814 V9.0.0, "Evolved Universal Terrestrial Radio Access (E-UTRA); Further advancements for E-UTRA physical layeraspects (Release 9)", 3rd Generation Partnership Project, Tech. Rep., 2010 [Online]. Available: http://www.3gpp.org. 
International Journal of Future Generation Communication and Networking

Vol. 10, No. 12 (2017) 